\title{
Microstructure and functional superelasticity property changes in thin NiTi wires heat treated by electric current
}

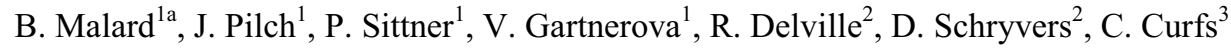 \\ ${ }^{1}$ Institute of Physics, Na Slovance 2, 18221 Praha, Czech Republic \\ ${ }^{2}$ EMAT, University of Antwerp, Groenenborgerlaan 171, B-2020 Antwerp, Belgium \\ ${ }^{3}$ ESRF, 6 rue Jules Horowitz 38043 Grenoble, France
}

\begin{abstract}
High energy synchrotron X-ray diffraction, transmission electron microscopy and mechanical testing were employed to investigate the evolution of microstructure, texture and functional superelastic properties of 0.1 $\mathrm{mm}$ thin as drawn Ni-Ti wires subjected to a nonconventional heat treatment by controlled electric current (FTMTEC method). As drawn Ni-Ti wires were prestrained in tension and exposed to a sequence of short DC power pulses in the millisecond range. The annealing time in the FTMT-EC processing can be very short but the temperature and force could be very high compared to the conventional heat treatment of SMAs. It is shown that the heavily strained, partially amorphous microstructure of the as drawn Ni-Ti wire transforms under the effect of the DC pulse and tensile stress into a wide range of annealed nanosized microstructures depending on the pulse time. The functional superelastic properties and microstructures of the FTMT-EC treated Ni-Ti wire are comparable to those observed in straight annealed wires.
\end{abstract}

\section{Introduction}

Shape memory alloys /SMA/ have attracted considerable attention for their unique thermo-mechanical functional properties deriving from the martensitic transformation /MT/ in solid state. Among them, the near equiatomic Ni-Ti alloy $[1,2]$ undergoes a MT from a high-temperature parent phase austenitic B2 [3] to a low-temperature monoclinic B19' martensitic phase [4]. It is one of the unique features of SMAs and particularly of Ni-Ti that the functional superelastic properties /FSP/ as well as the austenite shape of an element can be to a large extent set by the engineers. The procedure to achieve that is basically a heat treatment under mechanical constraint $[5,6]$. The Ni-Ti wires, sheets, tubes etc. are being produced from extruded semiproducts by multiple hot working passes finished by a final cold working. In this so called "cold worked" (as drawn) state, the alloy features a heavily deformed microstructure and does not show any FSP. This complex microstructure needs to be modified by a heat treatment into an annealed austenite microstructure in which the Ni-Ti element shows the desired FSP (Fig. 1). If the final heat treatment is performed under mechanical constraint, it is called "shape setting". Straight annealing treatment of $\mathrm{Ni}-\mathrm{Ti}$ wires is thus a special case of shape setting in which a constant force $\sim 20 \mathrm{MPa}$ is applied during the heat treatment. The shape setting has already been firmly established in the Ni-Ti technology. It is essential that the mechanical as well as FSP of the Ni-Ti (transformation temperatures, stresses, strains, yield stress, etc.) can be in large extent adjusted [5-9] by employing a proper combination of heat treatment condition before last cold working, amount of the cold work performed in the last wire drawing step, type of constraint, temperature and time of the heat treatment and post heat treatment thermo-mechanical cycling.

The effect of the heat treatment temperature and time on the transformation temperatures of Ni-Ti as investigated by DSC was extensively reported in the literature $[1,5]$. This now seems to be relatively well understood particularly for the Ni-rich Ni-Ti alloys commonly used to manufacture the superelastic medical NiTi wires. Depending on the alloy (chemical composition, hot work-texture, cold work) and desired engineering application, "best heat treatment conditions" can be found in the range of temperatures $400^{\circ} \mathrm{C}-500^{\circ} \mathrm{C}$ and treatment time 10-60 minutes. Less known is the role of the mechanical constraint applied during the shape setting. It affects the functional behaviors as clearly shown for example by Favier [10] for the case of thin walled $\mathrm{Ni}$-Ti tubes for stent production. We have got to work with thin Ni-Ti wires $(d=0.1 \mathrm{~mm})$ for application in smart textiles. These thin wires often exhibit excellent FSP [9] as shown in figure 1 (e.g. strength up to $2 \mathrm{GPa}$,

\footnotetext{
a email: benoit.malardesimap.grenoble-inp.fr
} 
superelasticity at stresses $\sim 1 \mathrm{GPa}$, superelastic window larger than $200^{\circ} \mathrm{C}$, good stability of cyclic deformation responses) probably due to the nanograin sized microstructure found in as drawn and straight annealed wires.

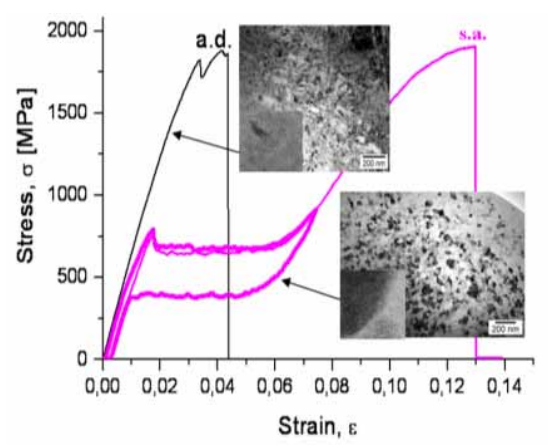

Fig. 1: Stress-strain response and HRTEM evidence on microstructure in as drawn (a.d.) and straight annealed (s.a.) $50 \mu \mathrm{m}$ thin superelastic NiTi wire

We employed various in-situ experimental techniques to follow evolution of cold worked microstructure during various heat treatments responsible for setting of the FSP and shape of Ni-Ti wires. The heat treatment was performed by pulsed DC electric current of millisecond pulse duration [6, 7, 8]. We have followed the evolution of electric power, temperature, tensile force, electric resistance of the treated wire with the heating time [6]. It turned out that the functional properties of the heat treated Ni-Ti wire can be very precisely controlled by selecting parameters of the electric power pulse and external force. This finally led to the development of a novel technique for Final Thermo-Mechanical Treatment of Ni-Ti wires by Electric Current /FTMT-EC/. A dedicated experimental equipment for continuous FTMT-EC treatment of long Ni-Ti filaments for textile applications was designed and built [7].

In this work, we present results of ex-situ high energy synchrotron X-ray diffraction and TEM investigations focused on microstructures and FSP of FTMT-EC treated Ni-Ti wires. The results together with in-situ investigations reported elsewhere $[6,8]$ helped significantly to get better understanding of the recovery processes responsible for microstructure changes and functional property setting in during the heat treatment.

\section{Experiments and Methods}

\subsection{Shape setting experiments}

Fort Wayne Metals superelastic cold worked Ni-Ti wire denoted as\#1 with diameter $\mathrm{d}=0.1 \mathrm{~mm}$ was used in this study. Stress-strain responses of this wire in cold work state and straight annealed by the company are shown in figure 1. The pulsed FTMT-EC experiment was carried out using a miniature deformation rig MITTER developed for efficient thermo-mechanical testing of FSP of thin Ni-Ti filaments for textile applications. The MITTER rig has $100 \mathrm{~N}$ load cell, electrically isolated grips, Peltier furnace, laser micrometer for strain measurement, special electronics allowing to send an electric power pulse with controlled shape in $\mu$ s range to heat the Ni-Ti wire to high temperatures, electric resistance measurement electronics and LabView controlled software.

In a typical in-situ shape setting experiment [6], the as drawn $\mathrm{Ni}-\mathrm{Ti}$ wire is mounted on the rig and its elastic response is first evaluated. After the initial length $l_{0}(\sim 50 \mathrm{~mm})$ and initial electrical resistance of the wire $\rho_{0}$ are evaluated, the wire is preloaded to given tensile stress at room temperature and its length is fixed. Following that, the length of the wire is kept constant while it is exposed to a controlled DC power pulse characterized by power $\mathrm{P}\left(\mathrm{P}_{\mathrm{HIGH}}\right)$ and parameters $\mathrm{t}_{1}, \mathrm{t}_{2}, \mathrm{t}_{3}$ (Fig. $\left.2 \mathrm{a}\right)$. As the temperature of the wire increases due to the supplied heat, the thermally activated recovery processes are triggered and proceed as long as the temperature is sufficiently high. The activity of the recovery processes is evidenced by the in-situ observed variation of force and electric resistance of the wire [6]. Temperature of the wire is calculated taking into account the Joule heat supply, temperature losses due to interaction with environment. After the shape setting treatment is finished, the superelastic response at room temperature is evaluated.

\subsection{Synchrotron X-ray diffraction}

\subsubsection{Introduction}

The X-ray beam delivered by third generation synchrotron sources is characterized by a high brilliance, a large spectral band, a small source size and divergence, polarization and a temporal structure. In present work, we report on ex-situ synchrotron $\mathrm{X}$-ray investigations on a $\mathrm{Ni}$-Ti wire performed as follows. The as drawn superelastic Ni-Ti wire $\left(l_{0}=50 \mathrm{~mm}\right)$ was preloaded to $100 \mathrm{MPa}$ and its length was fixed. After that, sequence of 
multiple successive electrical pulses $\left(P_{n}=125 \mathrm{~W} / 100 \mathrm{~mm}, \mathrm{t}_{1}=0.1 \mathrm{~ms}, \mathrm{t}_{2}=(0,1,2 \ldots 25 \mathrm{~ms})\right.$ and $\left.\mathrm{t}_{3}=0.05 \mathrm{~ms}\right)$ was sent to it (Fig. 2b). With this approach, the $25 \mathrm{~ms}$ pulse was in fact carried out in steps to allow the acquisition of high quality X-ray measurements after each pulse (2D diffraction pattern (Fig. 3a) was recorded for $20 \mathrm{~s}$ ). It is essential that peak temperature reached in each successive electric current pulse was slightly higher than in the preceding $1 \mathrm{~ms}$ shorter pulse (Fig. 2b). The evolution of stress and electrical resistance of the wire was followed in the experiment. This interrupted shape setting experiment was repeated again using another as drawn wire sample and FSP at room temperature were evaluated after each pulse instead of the X-ray measurements. These wires were used to prepare thin specimens for TEM studies.

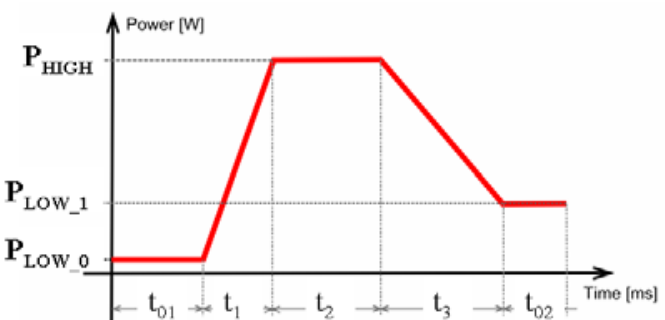

a)

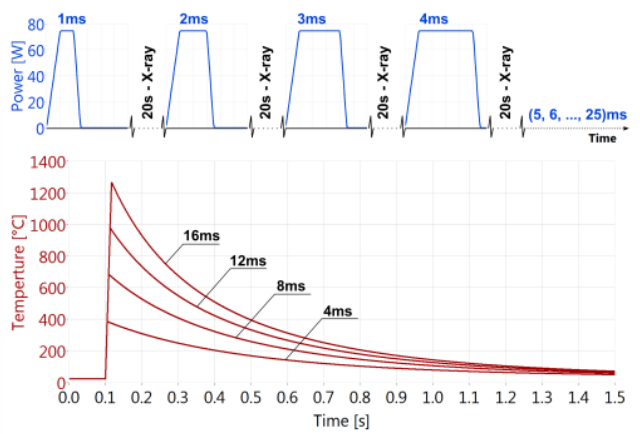

b)

Fig. 2: a) DC power pulse applied in the FTMT-EC treatment; b) Temperature evolution during the interrupted FTMT-EC treatment $\left(\mathrm{P}_{\mathrm{n}}=125 \mathrm{~W} / 100 \mathrm{~mm} / 0.1 \mathrm{~mm}, \mathrm{t}_{1}=0.1 \mathrm{~ms}, \mathrm{t}_{2}=(0,1,2 \ldots 25 \mathrm{~ms})\right.$ and $\left.\mathrm{t}_{3}=0.05 \mathrm{~ms}\right)$ employed in ex-situ X-ray experiment.

\subsubsection{Ex-situ synchrotron $X$-ray measurements}

A monochromatic X-ray beam with an energy of $45 \mathrm{keV}(\lambda \sim 0.2755 \AA)$ obtained using a double Laue monochromator on ID11 at ESRF was used. The beam is focused down to $150 \mu \mathrm{m}$ in vertical and horizontal directions by using RX lens [11]. The Frelon2K [12] was chosen as detection system and positioned at a distance enabling the measurement of diffraction peaks from a diffraction angle of 0 degree to the diffraction angle corresponding to the $\{211\}$ reflection of the austenite. The thin Ni-Ti wire sample prestrained in the MITTER rig was placed in vertical direction perpendicularly to the beam. The intensity of individual reflections observed in the 2D diffraction patterns (Fig. 3a) is not equally distributed in different directions due to the strong texture in the wire. Since the intensity observed along the vertical direction corresponds to lattice planes oriented with normals parallel to the wire axis we denote the three " $\pm 10^{\circ}$ integration cakes" in which the intensity was evaluated as axial at $90^{\circ}$, radial at $0^{\circ}$ and mid at $45^{\circ}$.

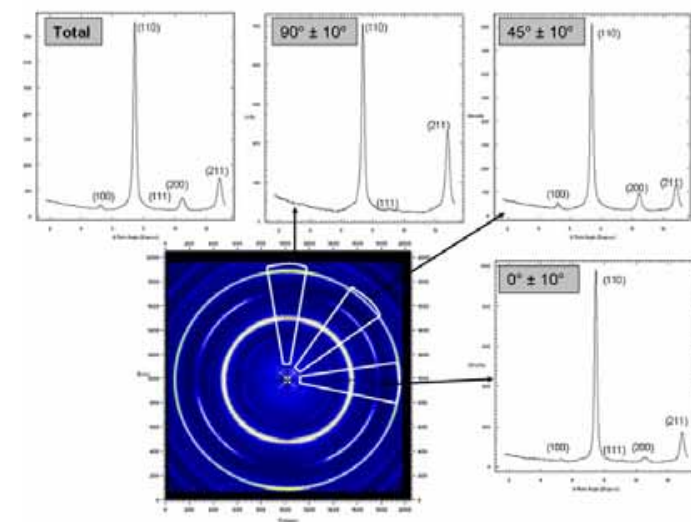

a)

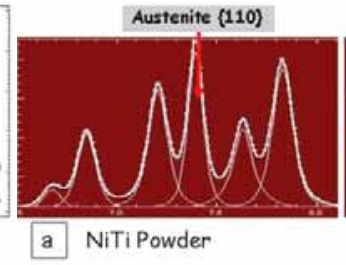

a NiTi Powder

Fig. 3: a) A diffraction pattern of FTMT-EC treated NiTi wire acquired on a $2 \mathrm{D}$ detector with superimposed three " $\pm 10^{\circ}$ integration cakes" in which the intensity was evaluated. The spectrum denoted as Total corresponds to $360^{\circ}$ integration; b) Diffraction results in the same experimental condition from a) NiTi powder b) NiTi wire before shape setting c) NiTi wire after shape setting.

The integration was also made for the entire whole rings. The austenite reflections were analyzed for intensity, Full Width at Half Maximum /FWHM/ and peak position /2theta/. In order to obtain a diffraction pattern of the intensity versus the diffraction angle, a calibration has to be performed. To do so, a very fine capillary of $300 \mu \mathrm{m}$ is covered of $\mathrm{LaB}_{6}$ powder and places at the Ni-Ti wire position. The obtained diffraction pattern was first corrected for spatial distortion using the fit $2 \mathrm{~d}$ software [13]. Then, the horizontal and vertical 
tilts of the detector were calculated, as well as the sample to detector distance. These numbers were used for the integration of each diffraction pattern, taken during the experiment, after spatial correction and subtraction of the dark field (which corresponds to the electronic noise of the CCD).

To obtain representative stress free spectra, Ni-Ti powder in a mixture of austenite and martensite phases at room temperature was placed instead of the $\mathrm{LaB}_{6}$ powder on the capillary and a diffraction pattern was taken. The diffraction pattern of the Ni-Ti powder is quite different from the diffraction pattern of the Ni-Ti wire (Fig. 3b) and gives much better resolution. This is due to the very complex microstructure of the Ni-Ti wires consisting of a mixture of heavily strained austenite, R-phase, martensite and amorphous phases with frozen in internal stresses. In this study, we followed the evolution of the $\{200\},\{110\}$ and $\{211\}$ austenite reflections by analyzing the integrated intensity, the position and the FWHM of each diffraction peak, using the LAMP software [14]. The full pattern was analyzed considering the austenite phase Pm-3m, the R-phase P-3[15] and the martensitic phase $\mathrm{P} 2{ }_{1} / \mathrm{m}$ with the GSAS software [16].

\subsection{Transmission electron microscopy observations}

Transmission electron microscopy /TEM/ studies were carried out to follow the evolution of microstructure with increasing pulse time of electric power pulse applied in the FTMT-EC treatment. The TEM samples were extracted from FTMT-EC treated Ni-Ti wires using the focused ion beam /FIB/ technique. The wires were cut and their cross-section polished with liquid diamond to obtain a smooth and even surface for the FIB extraction [17]. Slabs of $15 \mu \mathrm{m} \times 5 \mu \mathrm{m} \times 40 \mathrm{~nm}$ are extracted from the core of the wire in order to avoid the surface oxidation layer. Conventional transmission electron microscopy /CTEM/ observations were carried out in a Philips CM20 microscope operated at $200 \mathrm{kV}$ using a side-entry type double-tilt specimen holder with angular ranges of $+/-45^{\circ}$. All the selected area diffraction pattern presented in this paper were taken with a $0.5 \mu \mathrm{m}$ effective diameter aperture.

\section{Experimental Results}

\subsection{High energy synchrotron X-ray diffraction}

Figure 4 summarizes the three different kind of experimental results: 2D X-ray diffraction patterns, TEM images of microstructure, electron diffraction patterns and functional stress-strain responses of the NiTi wires FTMT-EC treated with selected pulse times. Let us first focus on the second column with $2 \mathrm{D}$ X-ray diffraction patterns. The broad continuous ring recorded on the $2 \mathrm{D}$ pattern of the as drawn untreated wire $(0 \mathrm{~ms})$ indicates the presence of mixture of austenite, martensite and amorphous phases. The longer is the applied electric pulse, the higher temperature was reached in the treatment and the finer are the austenitic rings. This suggests that with increasing pulse time, the martensite and amorphous phases disappear - transform or crystallize to the austenite phase and internal stresses are relaxed. The rings are segmented due to the wire texture. The segmentation is qualitatively similar for pulse times 0-16 ms but changes dramatically for longer pulses.

Finally, the point wise diffraction pattern recorded at $25 \mathrm{~ms}$ (Fig. 5a) suggest that most of the diffraction signal comes from fully recrystallized microstructure containing grains which are too big with respect to the analyzed volume $\left(\sim 0.1 \times 0.1 \mathrm{~mm}^{2}\right)$ and preferentially oriented with $<111>$ crystal directions along the wire axis. Since such treated wire does not show any superelasticity we did not investigate the microstructure by TEM in detail. Figure 6 shows the results of the diffraction pattern analyses as introduced in figure 3a which provide more detailed information. The integrated intensities, peak positions, and peak widths evaluated from the analyses of the $\{200\},\{110\}$ and $\{211\}$ austenite reflections are plotted against the pulse time. It can be seen that the intensity of the austenite peaks starts to increase only at pulse time $8 \mathrm{~ms}$ for all directions and reflections. But the peak positions and FWHM vary significantly in the $4-8 \mathrm{~ms}$ range. This is of particularly visible for the strongest $\{110\}$ reflection. This can be taken as evidence for relaxation of internal stresses and martensite to austenite transformation taking mainly place at these short pulse times. The residual stresses and martensite phase exist in the wire due to the final cold work.

For treatments 8-12 ms, we observe that the integral intensity steadily growing for all directions and reflections, the peak positions change and FWHM decreases significantly. In this range, the wire shows useful superelastic responses (Fig. 4). In the range of pulse times 12-16 ms, the intensity still increases though the trends are slightly different, the FWHM still decreases but the peak positions remain unchanged. This suggests that somehow different recovery processes take place at pulse times $12-16 \mathrm{~ms}$ and that there are no internal stresses anymore for pulse times longer than $12 \mathrm{~ms}$.

The 13-16 ms treated Ni-Ti wires still show superelasticity but accompanied by increasing irrecoverable strains suggesting significant plastic deformation due to a lower yield stress for plasticity of these wires.

At pulse times 16-20 ms, the integrated intensities evolve very differently than before and not monotonously. There are maxima of the integrated intensity in the 16-20 ms range depending on the integration cake orientation suggesting a strong change in grain size and/or texture in the treated wire. The wires treated 
with pulse time $\geq 18 \mathrm{~ms}$ do not show superelasticity. There is simultaneous forward transformation and plastic deformation on forward loading but no strain reversal and reverse transformation on unloading.

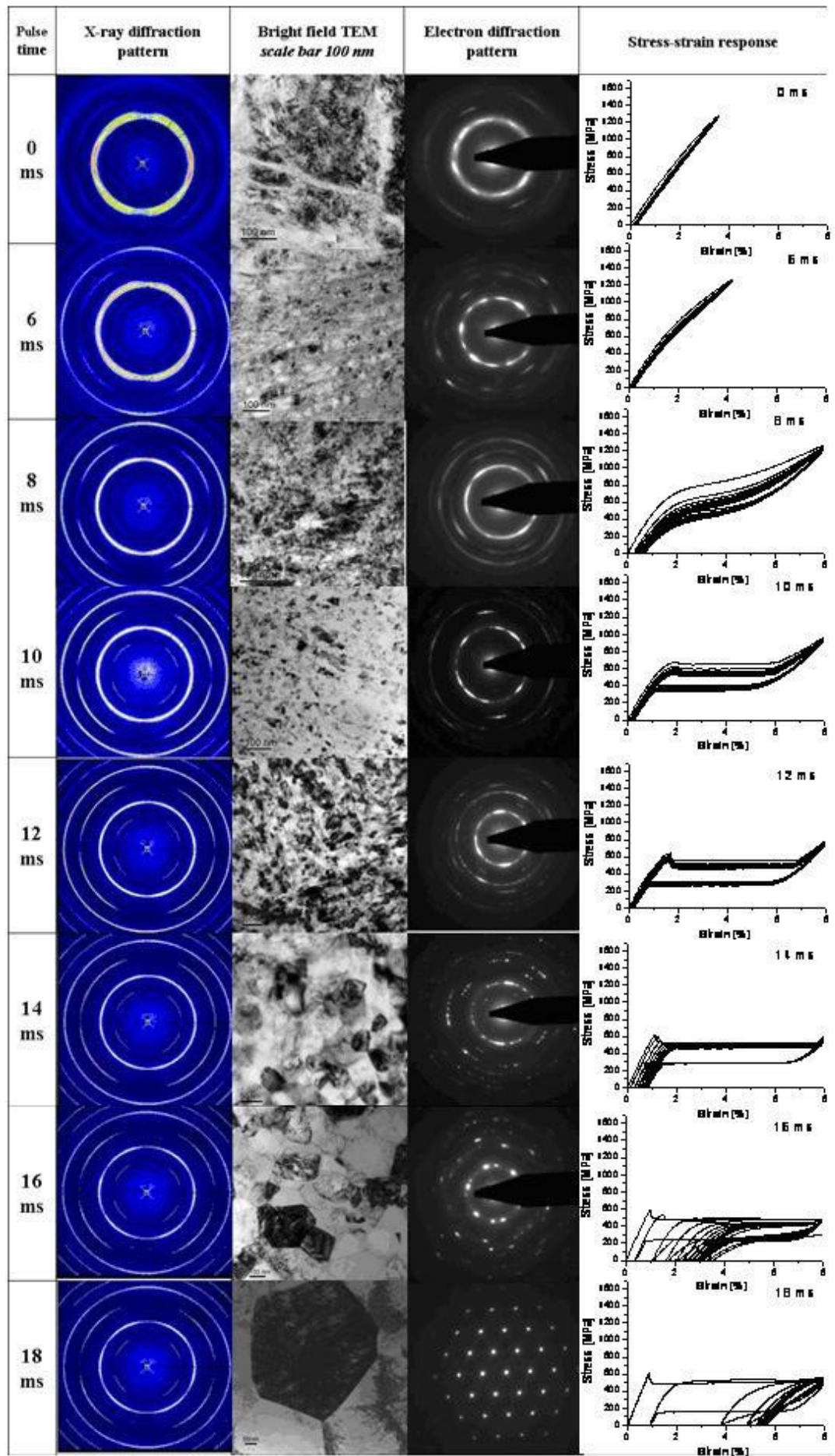

Fig. 4: Evolution of microstructure, texture and functional superelastic properties of superelastic $0.1 \mathrm{~mm}$ thin $\mathrm{Ni}$-Ti wire exposed to the FTMT-EC treatment with increasing pulse times.

In addition, qualitative information about the character of the internal stress can also be deduced from the evolution of peak positions in the 4-12 ms range. Since the peak position decreases in radial direction but increases in the axial direction, the lattice spacing for lattice planes with normals perpendicular (parallel) to the wire axis increases (decreases). This means that the internal stress frozen in after cold work is tensile and becomes annealed out at pulse time of about $12 \mathrm{~ms}$, for which the wire shows "best" superelastic properties. The interpretation of the observed decrease of FWHM increasing pulse time in the range 4-16 ms is difficult since the reflections become possibly narrower due to multiple phenomena. These phenomena are the increasing size of nanograins, the annihilation of crystal defects, the annealing out of the internal stresses in the newly forming 
austenite phase and the coalescence of the neighbouring nanograins. FWHM decrease is terminated at pulse time $\sim 16 \mathrm{~ms}$ and stabilizes at $\mathrm{FWHM}=0.05^{\circ}$. This means that there are neither internal stresses nor lattice defects induced by cold work after $16 \mathrm{~ms}$ treatment and that the significant grain growth up to several $\mu \mathrm{m}$ range (Fig. 4) does not affect the FWHM anymore.

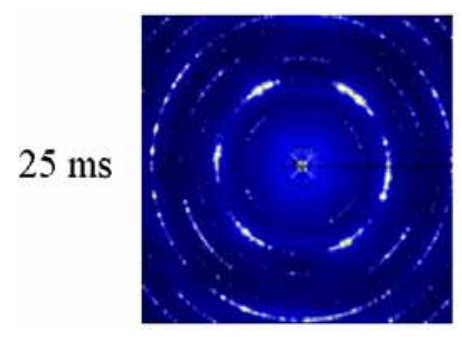

a)

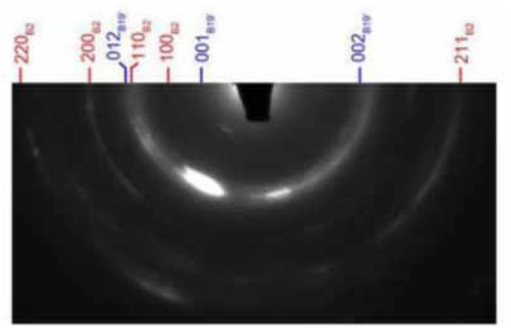

b)

Fig.5: a) X-ray diffraction pattern recorded at $25 \mathrm{~ms}$ pulse time; b) The analysis of the ring pattern of the $0 \mathrm{~ms}$ sample reveals the presence of B2 austenite and a small amount of B19' martensite in addition to the amorphous diffuse rings

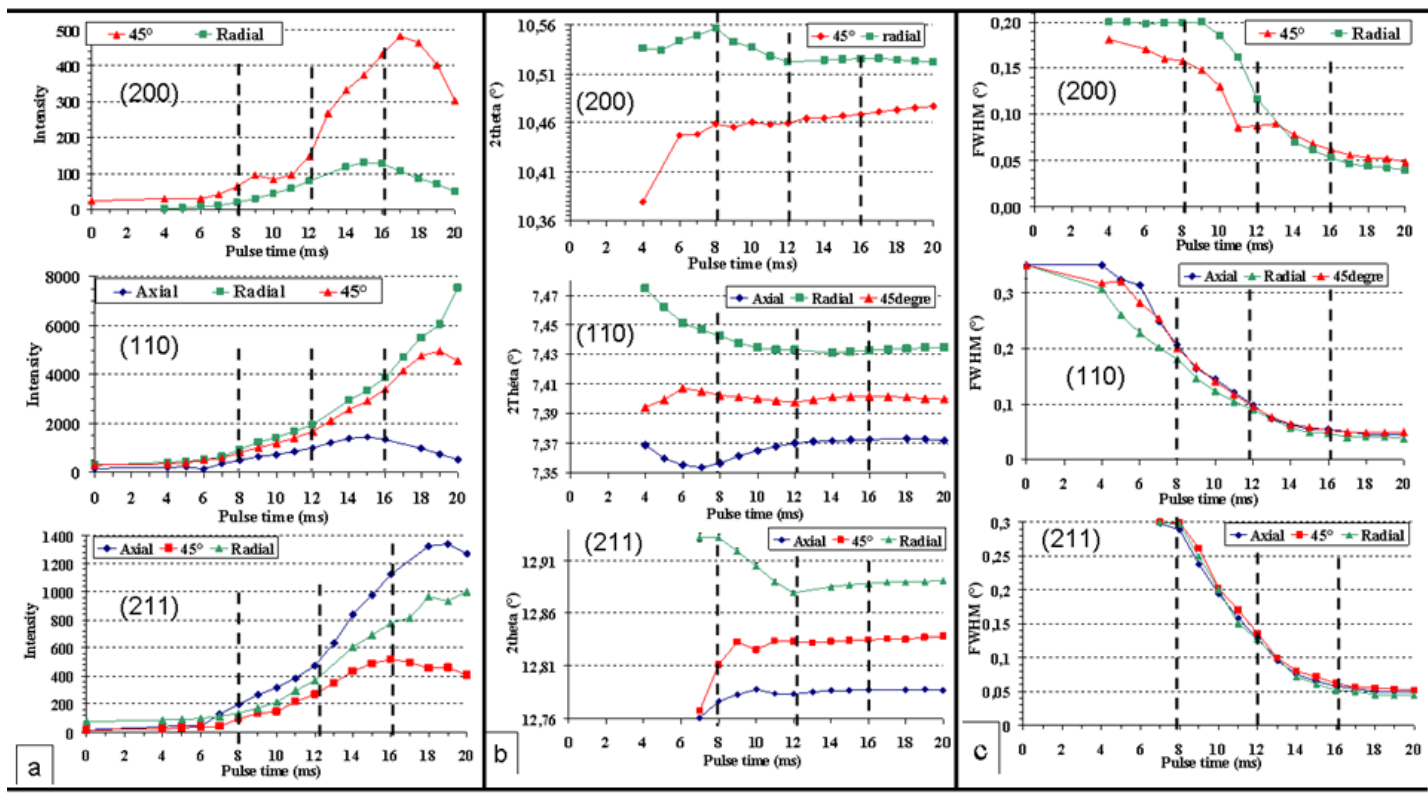

Fig. 6: Evolution of a) integrated intensity; b) peak position; c) FWHM of (200), (110) and (211) austenite reflections with increasing pulse time determined by the analysis of the ex-situ synchrotron X-ray results.

\subsection{Transmission electron microscopy investigations}

The composition of wires as determined by atomic absorption spectroscopy is 50.8 at.\%Ni -49.2 at.\%Ti which is in good agreement with the composition given by the manufacturer $(50.9$ at.\%Ni -49.1 at.\%Ti). Figure 4 shows a bright field electron micrograph of the as-received microwire (noted $0 \mathrm{~ms}$ ), which contains a high density of tangled dislocations [1]. The corresponding diffraction patterns in figures 4 and $5 \mathrm{~b}$ exhibit arched diffuse spots resulting from the presence of strains due to high density of dislocations and a diffuse halo ring, indicating the presence of an amorphous phase. A closer look at the indexed diffraction pattern in figure $5 \mathrm{~b}$ shows that the microstructure consists of a mixture of austenite, martensite and amorphous states. Signal from the martensite B19' is unambiguously identified by the (001)B19' reflection. Both (012)B19' and (002)B19' reflections are also visible but often merge with neighboring $\{110\}_{\mathrm{B} 2}$ reflection. The weak intensity of the B19' reflections suggests that only a very small amount of the phase is present. Since the Ms is below room temperature for this Ni-rich composition, it is obvious that the retained martensite must have been created and stabilized by severe plastic deformation during cold working.

Figure $4(0 \mathrm{~ms})$ shows also white stripes repeatedly found throughout the FIB slab. Similar amorphous bands have been reported for heavily cold-rolled Ni-Ti alloys [18-20]. The microstructure (Fig. 4 (6 ms)) consists of nanocrystals formed during polygonization of the severely deformed cold worked microstructure. The corresponding diffraction patterns show sharper diffraction spots indicating a strain relief due to the lattice recovery. The recovery processes involving polygonization-rearrangement or annihilation of dislocations [1] evidently take place in the 6 and $8 \mathrm{~ms}$ treated samples. A competing mechanism for the formation of new grains would be recrystallization but we believe that it occurs at pulse times equal to $12 \mathrm{~ms}$ and longer, where smaller recrystallized equi-axed grains can be clearly identified. For the $6 \mathrm{~ms}$ and above, diffraction patterns no longer 
show B19' reflections, indicating that the retained martensite was transformed to austenite. As the microstructure recovers, the functional properties are improved but a clearly defined superelastic loop is only obtained from 10 ms. Evidences of recrystallisation which is characterized by equi-axed, strain-free new grains were found in the $10 \mathrm{~ms}$ sample which still contains an important part of recovered microstructure. The $12 \mathrm{~ms}$ sample is mostly recrystallized with an average grain size of $50 \mathrm{~nm}$. Nearly completed recrystallization is also confirmed by the diffraction ring composed of randomly oriented crystalline reflections. A few pockets of recovered microstructure were found. They are characterized by a high strain contrast and by low angle boundaries between the recovered sub-grains.

At 14-16-18 ms pulse, the microstructure is similar but the average grain size has grown significantly (100-350-1000 nm). The stress-strain response is still superelastic, though increasing accumulation of irreversible plastic strains during cycling with increasing pulse time becomes a significant drawback.

Above $18 \mathrm{~ms}$ pulse, the austenite grains have grown up to a micrometer size and their disorientations are large as common for conventional recrystallized microstructure in NiTi. A selected area diffraction pattern taken from the central grain in the bright field image in figure $4(18 \mathrm{~ms})$ shows a $<111>$ single crystal diffraction. In addition, precipitates were frequently found in the $18 \mathrm{~ms}$ treated sample. A more detailed TEM study of the microstructures and precipitates will be published in a separate article [19].

\section{Discussion}

The heavily strained nanosized microstructure reported above appears evidently due to severe plastic deformation /SPD/ occurring during processing the Ni-Ti wire by multiple hot/cold work annealing passes. It has already been reported in the literature that $\mathrm{Ni}$-Ti can be relatively easily processed by various SPD methods (rolling [18, 19, 21], ECAP [20]) so it becomes partially or even completely amorphous [22]. The novel feature reported in this manuscript is that similar partially amorphous microstructure exists also in commercial superelastic thin $\mathrm{Ni}$-Ti wires and that this microstructure can be manipulated in a very controlled manner by the FTMT-EC treatment so that the wire is shape set and the required FSP of the wire are obtained. In both conventional straight annealing and FTMT-EC treatments, the wire is exposed to high temperature and uniaxial tension during the treatment. Nevertheless, there are major differences between these two methods.

While in the straight annealed case the wire is exposed to stresses of about $20 \mathrm{MPa}$ and temperature $400-500^{\circ} \mathrm{C}$ for about 10 minutes, in case of the FTMT-EC treatment (e.g. $125 \mathrm{~W} / 12 \mathrm{~ms}$ ), the temperature and mechanical stress in the wire reached values as high as $900^{\circ} \mathrm{C}$ and $700 \mathrm{MPa}$ for a very short time (Fig. 2b and 7a). The physical processes leading to the lattice recovery via microstructural changes during the heat FTMT-EC and conventional treatments thus proceed on completely different time scales and under different external forces. Figure 7a shows the response of the cold worked NiTi wire in the direct FTMT-EC pulse treatment (prestrain $340 \mathrm{MPa}$ ). Note that, in spite of the temperature increasing during the pulse, the electrical resistivity of the wire decreases nearly $40 \%$. This is because the effect of the evolution of the microstructure taking place during the treatment on the electrical resistance is even stronger than the effect of the increasing temperature. Although the tensile stress, temperature and electrical resistivity evolve very fast during the time $t_{2}$ of the DC pulse, the system electronics keeps the prescribed supplied power constant. Superelastic stress-strain curve at room temperature of the directly FTMT-EC treated cold worked NiTi wire with superimposed electric resistance-strain in-situ response suggesting R-Phase activity is shown in figure 8a.

The second issue making the FTMT-EC treatment different from the conventional straight annealing in a furnace is the possible direct action of electrons on the recovery processes. Indeed, there are reports in the literature suggesting that passing electrons affect plastic deformation - electroplasticity [23, 24] defect mobility [25], crystallization [26] processes in solids.

A third issue making a difference is the possible formation of the surface oxide layers which are obviously different for both methods. Finally, there are concerns about the homogeneity of the temperature in the FTMT-EC processed wire and its effect on the possible gradient of microstructure from the centre to the surface of the wire. Since the heat comes from inside the material treated by the electric current, the highest temperature in the FTMT-EC treated wire should be indeed expected in its centre. Nevertheless, considering the poor heat transfer into air across the wire surface and quasiadiabatic conditions for rather short pulses, we assume a homogeneous temperature distribution, particularly during the heating branch when maximal temperatures are reached.

Let us briefly comment on the microstructure, texture and FSP evolutions with increasing pulse time. Recall that FTMT-EC treatment was purposely performed (for the sake of the X-ray measurements) via the interrupted pulse treatment introduced in section 3.1. That means that the wire denoted as $12 \mathrm{~ms}$ in figure 4 was in fact treated by sequence of pulses $(1+2+3+\ldots+12 \mathrm{~ms})$. The in-situ response in the last $12 \mathrm{~ms}$ pulse of the interrupted treatment is shown in figure $7 \mathrm{~b}$. In this case, the electrical resistance of the wire increases with increasing temperature during the pulse. This is because the starting microstructure after previous $1-11 \mathrm{~ms}$ pulse treatment is quite different from the cold worked starting microstructure in figure $7 \mathrm{~b}$. Note the much lower starting electrical resistivity value in figure $7 \mathrm{c}$ compared to figure $7 \mathrm{a}$. In the interrupted treatment, the wire was 
exposed to high temperatures for time much longer than $12 \mathrm{~ms}$. Nevertheless, the effects of the interrupted and direct treatments on the superelastic stress-strain response of the Ni-Ti wire are nearly the same (Fig. 7c). The electrical resistivity of the wire measured at room temperature after both treatments are nearly the same too (Fig. $7 \mathrm{a}$ and $7 \mathrm{~b}$ ). This suggests that similar microstructures were created by direct and interrupted $12 \mathrm{~ms}$ pulse treatments. The direct and interrupted pulse treatments are thus considered to be nearly equivalent in their effects on the FSP of the wire. Hence, the presented combined X-ray and TEM results can be considered representative also for direct FTMT-EC pulse treatments.

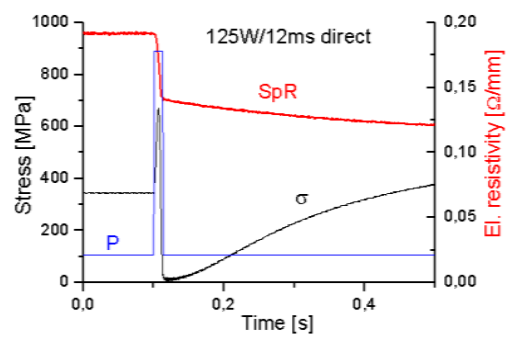

a)

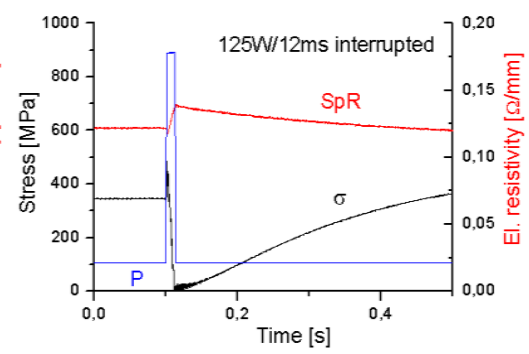

b)

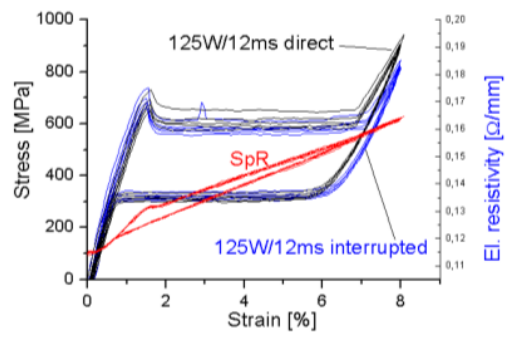

c)

Fig. 7: In-situ record of supplied power P, stress response $\sigma$, and electrical resistivity SpR during direct FTMT-EC treatment $(125 \mathrm{~W} / 12 \mathrm{~ms})$ a) one $12 \mathrm{~ms}$ pulse treatment; b) the starting microstructure of the wire was not the cold worked one but already received $1,2 \ldots 11 \mathrm{~ms}$ pulse treatment; c) Superelastic stress-strain response (10 cycles at RT) of NiTi wires treated by direct and interrupted pulse treatments. Electrical resistivity $(\mathrm{SpR})$ responses during the stress-strain tests are nearly identical

Table 1: Characteristics of the microstructure and superelastic functional properties of FTMT-EC treated superelastic NiTi wire.

\begin{tabular}{|c|c|c|c|c|c|}
\hline \multirow{2}{*}{ Pulse time } & \multicolumn{3}{|c|}{ Microstructure } & \multicolumn{2}{c|}{ Functional Properties } \\
\cline { 2 - 6 } & $\begin{array}{c}\text { SpR } \\
{[\Omega \mathrm{mm}]}\end{array}$ & $\begin{array}{c}\text { Grain size } \\
{[\mathrm{nm}]}\end{array}$ & $\begin{array}{c}\text { Fract. of aust. } \\
{[\%]}\end{array}$ & $\begin{array}{c}\text { Transformation strain } \\
{[\%]}\end{array}$ & $\begin{array}{c}\text { Unrecovered strain } \\
{[\%]}\end{array}$ \\
\hline $0 \mathrm{~ms}$ & 0.19 & - & 85 & 0 & 0.1 \\
\hline $4 \mathrm{~ms}$ & 0.177 & - & 85.4 & 0 & 0.1 \\
\hline $6 \mathrm{~ms}$ & 0.162 & $5-10$ & 86.2 & 3 & 0.2 \\
\hline $8 \mathrm{~ms}$ & 0.135 & $10-20$ & 87.5 & 4.5 & 0.7 \\
\hline $10 \mathrm{~ms}$ & 0.124 & $20-40$ & 88.8 & 6 & 0.28 \\
\hline $\mathbf{1 2 m s}$ & $\mathbf{0 . 1 1 9}$ & $25-50$ & $\mathbf{9 0 . 5}$ & 6.5 & 0.85 \\
\hline $14 \mathrm{~ms}$ & 0.113 & $70-110$ & 94 & 7 & 3.17 \\
\hline $16 \mathrm{~ms}$ & 0.113 & $200-700$ & 97.9 & 7 & 5.6 \\
\hline $18 \mathrm{~ms}$ & 0.113 & $800-1200$ & 100 & & \\
\hline
\end{tabular}

The texture in the as drawn Ni-Ti wire evidenced by the segmented 2D X-ray diffraction patterns (Fig. 4) was created by the sequence of many multiple cold/hot working passes, not just in the final cold drawing step. The reason why it does not evolve very much with increasing pulse time up to $12 \mathrm{~ms}$ (16 ms), even if the microstructure changes drastically, is probably related to the fact that the newly crystallized nanograins approximately maintain the original crystal lattice orientation and hence the original texture. At largest pulse times above $25 \mathrm{~ms}$, the grains are too large for reliable assessment of thin wire texture from the X-ray diffraction pattern. Nevertheless, it can be deduced from figure $5 \mathrm{a}$ that the $25 \mathrm{~ms}$ pulse treated wire contains large grains preferably oriented with $<111\rangle$ crystal direction along the wire axis (see the $\langle 100\rangle$ diffraction intensity distributed at $\sim 35^{\circ}$ from vertical direction and even some $<111>$ intensity (forbidden in the B2 structure) along vertical axis).

Key issue for the development of the FTMT-EC method is to know the relationship between the characteristics of the applied DC pulse, mechanical constraints and FSP of the treated wires. Since the FSP reflect the microstructures in the wires, the key to the understanding of this relationship are the microstructures. Table 1 summarizes available experimental information on the microstructures and FSP for the FTMT-EC treatments presented in figure 4. Electrical resistivity SpR (as measured in-situ during the X-Ray experiment), grain size (as evaluated from TEM images) and volume fraction of austenite phase (from X-ray data) characterize the microstructures, while transformation strain and accumulated irreversible strain in 10 superelastic cycles at room temperature characterize essential features of the superelasticity of the wire. Electrical resistivity of the wire decreases as it follows the microstructure evolution with the increasing pulse time (Fig. 8).

Another microstructure characteristic is the internal stress (2theta) in the wire. Qualitatively, its evolution with pulse time can be inferred from figure 6 middle (its decrease with pulse time saturates at $12 \mathrm{~ms}$ ). Quantitative evaluation of internal stress from X-ray studies, however, appeared to be problematic There is not enough space for detailed discussion, this can be found in related papers focusing the directly the in-situ X-Ray 
studies. Regarding the FSP (table 1), surprisingly, the upper plateau stress and stress hysteresis width in the first cycle are not significantly affected by the pulse time. Cyclic stability of the superelastic loop, however, depends on the pulse time significantly. Best cyclic stability was observed for treatments with $\sim 12 \mathrm{~ms}$ pulses, where the plateau strains reached $6.5 \%$, localized deformation evidenced by the sharp yield point starts to appear and Rphase transformation precedes the martensitic one upon cooling and mechanical loading.
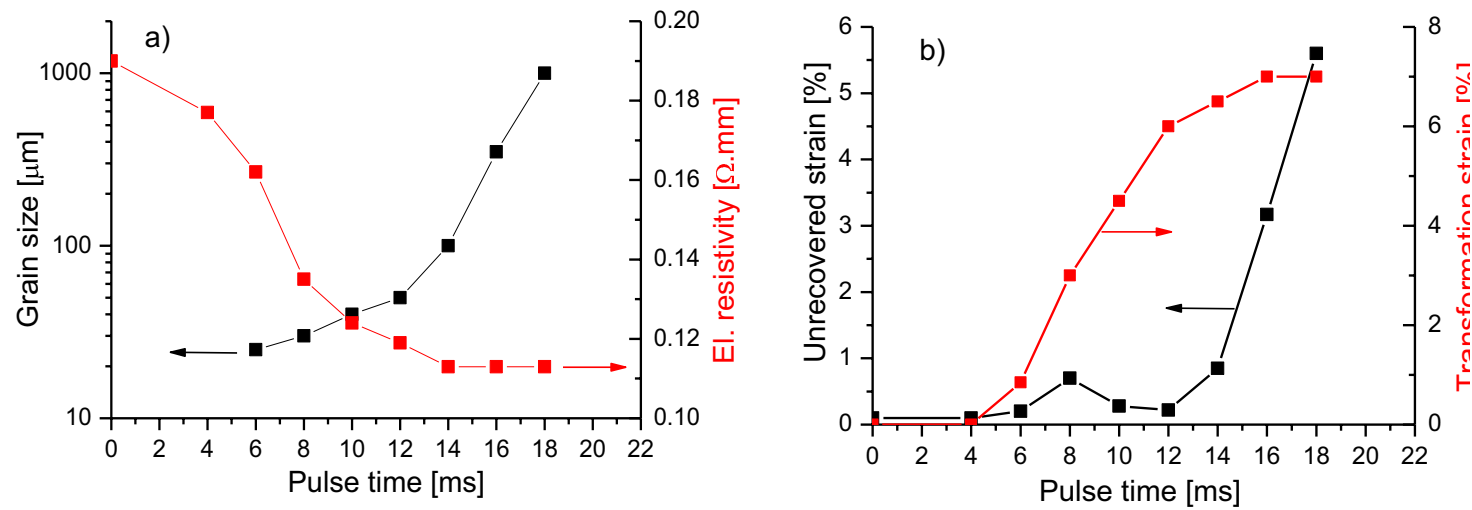

Fig. 8: Grain size (a), electrical resistivity (a) transformation strain (b) and unrecovered strain (b) of the FTMT-EC treated NiTi wires in dependence on the pulse time during the interrupted treatment.

In fact, similar microstructures and FSP were achieved by conventional straight annealing treatment (Fig. 1) and by pulsed electric current $125 \mathrm{~W} / 12 \mathrm{~ms}$ under mechanical constraint $100 \mathrm{MPa}$ (Fig. $7 \mathrm{c}$ ) although wire diameters are different. This microstructure optimal for superelasticity is almost fully crystalline austenitic, mean grain size is $\sim 50 \mathrm{~nm}$, still contains some defects but no or very little retained martensite phase and very little residual stress due to preceding cold work.

\section{Conclusion}

High energy synchrotron X-ray diffraction, TEM and mechanical testing were employed to investigate evolution of the nanograin sized microstructure, texture and functional superelastic properties of $0.1 \mathrm{~mm}$ thin as drawn superelastic Ni-Ti wires subjected to nonconventional heat treatment by controlled electric current (FTMT-EC method).

It is shown that the Ni-Ti wires can be treated with the FTMT-EC method in millisecond time interval in such a way that precise control of functional superelastic properties is achievable. A relationship among FTMT-EC processing parameters (DC power $125 \mathrm{Watts} / 100 \mathrm{~mm}$, preload $100 \mathrm{MPa}$, pulse time 1-25 ms), microstructure parameters (grain size, volume fraction of austenite) and functional superelastic properties (transformation strain, accumulated irreversible strain in 10 superelastic cycles) of treated NiTi wire were established.

Best superelastic response of the $0.1 \mathrm{~mm}$ thin $\mathrm{Ni}$-Ti wire was achieved by the FTMT-EC treatment using power $125 \mathrm{Watts} / 100 \mathrm{~mm}$, preload $100 \mathrm{MPa}$, and $12 \mathrm{~ms}$ pulse time. The corresponding microstructure is almost fully austenitic; means grain size is $\sim 50 \mathrm{~nm}$, still contains some defects but no or very little retained martensite phase and no residual stress due to preceding cold work.

\section{Acknowledgement}

The authors acknowledge the support of this work by Marie-Curie RTN MULTIMAT (MRTN-CT-2004-505226) as well as the support of ESRF for performing the synchrotron experiment (MA-358), support by EC via FP6 integrated projects AVALON (NMP2-CT-2005-515813), UPWIND (019945(SES6)) and project AV0Z10100520.

\section{References}

[1] K. Otsuka and X. Ren, Pr. Mat. Sci., 50, pp.511-678, (2005)

[2] H. Funakubo, Ed., Shape Memory Alloys (Gordon and Breach Science Publishers, New York, 1987)

[3] F.E. Wang, W.J. Buehler and S.J. Pickart, J. Appl. Phys. 36, 3232, (1965)

[4] Y. Kudoh et al., Acta. Metall., 33(11), pp.2049-2056, (1985)

[5] T.W. Duerig, K.N. Melton, D. Stöckel, and C.M. Wayman, eds., Engineering Aspects of Shape Memory

Alloys (Butterworth-Heinemann Ltd., London), 1990.

[6] J. Pilch, L. Heller, P. Sittner, in this volume

[7] Czech patent application PV 2009-279

[8] B. Malard et al., (in preparation)

[9] P. Šittner et al., Proc. of SMST 2006, Asilomar, California,USA., pp.553-568, (2008)

[10] D. Favier et al., Mater. Sc. and Eng. A 429 pp.130-136, (2006) 
[11] A. Simionovici, C. Schroer and B. Lengeler, X-ray Spectrometry: Recent Technological Advances, ISBN 047148640X, 9780471486404; pp.111-132, (2004)

[12] J-C. Labiche et al., Review of scientific instruments, 78, 091301, (2007)

[13] http://www.esrf.eu/computing/scientific/FIT2D/

[14] W.I.F. David et al., J. Appl. Cryst., 39, pp.910-915, (2006)

[15] D. Schryvers and P.L. Potapov, Mat. Trans. JIM 43 (5), pp.774 -779, (2002)

[16] http://www.ill.fr/data treat/lamp/front.html

[17] R. Delville et al., (in preparation)

[18] A.K. Srivastava, D. Schryvers and J. Van Humbeeck, Intermetallics 15, pp.1538-1547, (2007)

[19] J. Koike and D.M. Parkin, J. Mater. Res., 5(7), (1990)

[20] A.V. Sergueeva et al., Mater. Sc. and Eng. A, 339, pp.159-165, (2003)

[21] V. Brailovski et al., Mater. Trans., 47, pp.1-10, (2006)

[22] K. Inaekyan et al., J. Alloys and Compounds 473, Issues 1-2, pp.71-78, (2009)

[23] G.V. Stepanov and A.I. Babutskij, Strengths of materials 24, pp.310-315, (2002)

[24] A.M. Roschupkin and I.I. Bataronov, Russian Physics Journal, 39, pp.230-236, (1996)

[25] J.E. Garay et al. Applied Physics letters, 85, pp.573-575, (2004)

[26] M. Gao et al., Mater. Sc. and Eng. A, 337, pp.110-114, (2002) 